\title{
LISe Pixel Detector for Neutron Imaging
}

Elan Herrera $^{1}$, Daniel Hamm ${ }^{1}$, Brenden Wiggins ${ }^{2,3}$, Rob Milburn ${ }^{1}$, Arnold Burger ${ }^{3,4}$, Hassina Bilheux ${ }^{5}$, Louis Santodonato ${ }^{6}$, Ondrej Chvala ${ }^{1}$, Ashley Stowe ${ }^{1,2,3}$, and Eric Lukosi ${ }^{1}$

${ }^{1}$ Department of Nuclear Engineering, University of Tennessee, Knoxville, TN, USA

${ }^{2}$ Technology Development, Y-12 National Security Complex, Oak Ridge, TN, USA

${ }^{3}$ Department of Physics and Astronomy, Vanderbilt University, Nashville, TN, USA

${ }^{4}$ Department of Life and Physical Sciences, Fisk University, Nashville, TN, USA

${ }^{5}$ Chemical and Engineering Materials Division, Oak Ridge National Laboratory, Oak Ridge, TN, USA

${ }^{6}$ Instrument and Source Division, Oak Ridge National Laboratory, Oak Ridge National Laboratory, Oak Ridge, TN, USA

KEYWORDS: ${ }^{6} \mathrm{LiInSe}_{2}$; LISe; Neutron detection; Neutron imaging; Pixel detector; Semiconductor detector.

\section{AbStraCt}

Semiconducting lithium indium diselenide, ${ }^{6} \mathrm{LiInSe}_{2}$ or LISe, has promising characteristics for neutron detection applications. The $95 \%$ isotopic enrichment of ${ }^{6} \mathrm{Li}$ results in a highly efficient thermal neutronsensitive material. In this study, we report on a proof-of-principle investigation of a semiconducting LISe pixel detector to demonstrate its potential as an efficient neutron imager. The LISe pixel detector had a $4 \times 4$ of pixels with a $550 \mu \mathrm{m}$ pitch on a $5 \times 5 \times 0.56 \mathrm{~mm}^{3}$ LISe substrate. An experimentally verified spatial resolution of $300 \mu \mathrm{m}$ was observed utilizing a super-sampling technique.

\section{INTRODUCTION}

Cold neutron radiography and tomography comprise powerful detection tools for investigating both natural and engineered materials. Its sensitivity for real-space imaging relies on the total neutron interaction cross section with the material under investigation, which includes both scattering (neutronnuclei and neutron-lattice) and absorption [1]. Neutron imaging shares many similarities with X-ray imaging systems, albeit the mode of interaction of the interrogating beam is different. The comparison between these two imaging techniques is outside the scope of this study, but many references are available [1-3].

In the advancement of the capabilities of neutron imaging facilities, it has been indicated that there is a need for advances in suitable detection systems to reach the full potential of current and future neutron facilities, such as the Spallation Neutron Source (SNS) at Oak Ridge National Laboratory (ORNL). Specifically, a simultaneous enhancement of the detection efficiency (ideally 100\%), spatial resolution (less than $10 \mu \mathrm{m}$ ), large signal-to-noise ratio, (good gamma-ray rejection capability), and high temporal resolution (less than $1 \mu \mathrm{s}$ ) is desired [4]. Achieving the desired performance metrics outlined is a challenging prospect, but the use of a semiconductor-based imaging plane may provide the necessary performance. 
Current semiconductor-based cold neutron imagers rely on the use of a converter layer on a semiconductor detector $[1,5,6]$. The converter material is enriched with an isotope exhibiting large neutron capture cross section at thermal/cold energies, commonly ${ }^{6} \mathrm{Li},{ }^{10} \mathrm{~B}$ or ${ }^{157} \mathrm{Gd}$. The range of secondary charged particles in the converter layer, however, result in a few percent detection efficiency for thermal neutrons using ${ }^{10} \mathrm{~B}$ and ${ }^{6} \mathrm{LiF}$ converter layers [7]. Further, transport of energetic charged particles may result in a decreased energy resolution $[5,6]$. Using ${ }^{157} \mathrm{Gd}$ or ${ }^{113} \mathrm{Cd}$, which both have neutron capture cross sections an order of magnitude or more than ${ }^{10} \mathrm{~B}$ and ${ }^{6} \mathrm{Li}$, can result in efficiencies up to $40 \%$ [8]. Further, Gd- and Cd-based detection systems have the potential for high-resolution imaging because of the lower energy and range of the emitted particles in the neutron reaction compared to ${ }^{6} \mathrm{Li}$ and ${ }^{10} \mathrm{~B}$, limited only by the pixel density and pitch of the sensing substrate. However, the low energy of conversion electrons and x-rays emitted from ${ }^{\text {nat }} \mathrm{Gd}$ and ${ }^{113} \mathrm{Cd}$ results in a poor signalto-noise ratio and are subject to image contamination from internal and external gamma-rays. Imaging contamination from gamma-rays using ${ }^{10} \mathrm{~B}$ and ${ }^{6} \mathrm{LiF}$ coated sensors is also an issue, where higher thresholds may be required to minimize image contamination, resulting in reduced detection efficiency $[5,6]$. Therefore, although neutron imaging systems using semiconductors have been investigated, the low efficiency and/or poor signal-to-noise ratio have commonly led to the use of other types of detection systems, which have their own limitations of the achievable spatial resolution, neutron detection efficiency, and temporal resolution. For further discussion on different types of neutron imaging systems, the reader is referred to these excellent references [1, 3, 9-12].

Lithium indium diselenide $\left({ }^{6} \mathrm{LiInSe}_{2}\right.$ or $\left.\mathrm{LISe}\right)$ is a relatively new $\mathrm{A}^{\mathrm{I}} \mathrm{B}^{\mathrm{III}} \mathrm{X}_{2}^{\mathrm{VI}}$ type semiconductor with a band gap of $2.8 \mathrm{eV}$ [13]. It possesses a $24 \%{ }^{6} \mathrm{Li}$ concentration within the regular stoichiometry of the crystal, eliminating the need for separated conversion and detection layers. The detection of neutrons by LISe relies on the ${ }^{6} \mathrm{Li}\left(\mathrm{n},{ }^{3} \mathrm{H}\right){ }^{4} \mathrm{He}$ reaction, and the large $4.78 \mathrm{MeV}$ Q-value of the reaction provides an intrinsic potential for excellent neutron/gamma discrimination. The mean free path of thermal neutrons in LISe is $920 \mu \mathrm{m}$, enabling the use of thin LISe substrates for potentially efficient neutron detection. With these attributes, LISe may provide the desired sensor system attributes previously outlined. This report presents on the development and evaluation of a proof-of-principle LISe pixel detector to investigate its potential for cold neutron radiography.

\section{Materials ANd Methods}

\subsection{Neutron Imaging Sensor Development}

The LISe pixel detector was prepared by the synthesis method of Tupitsyn et al. [13] using lithium enriched in ${ }^{6} \mathrm{Li}$ to 95 at\%. A $5 \times 5 \times 0.56 \mathrm{~mm}^{3}$ yellow LISe crystal was used as the sensor substrate because they typically exhibit the best semiconducting properties (see Fig. 1) [14]. Substrate processing and LISe pixel detector fabrication took place at the Micro-Processing Research Facility at the University of Tennessee [15]. A 4x4 pixel pattern with a pitch of $550 \mu \mathrm{m}$ and a $100 \mu \mathrm{m}$ guard ring was deposited on the LISe sensor substrate using negative liftoff photolithography, as shown in Fig. 2 [16]. Indium was deposited via RF magnetron sputtering. Following successful patterning, the pixelated LISe sensor substrate (LISe pixel detector) was mounted to a custom sensor board using electronic grade silver paste and gold wire wedge bonding (see Fig. 3) [17].

The front-end electronics was designed using CadSoft EAGLE PCB Design Software ${ }^{\mathrm{TM}}$. The preamplifier circuit consists of twin 8-channel boards, where each board has its own filtered highvoltage line to bias the LISe pixel detector. Each pixel, or channel, from the sensor board was ACcoupled to Cremat ${ }^{\circledR}$ CR-110 (rev. 2) charge sensitive preamplifiers. A central DC power supply powered the 16 Cremat CR-110 (rev. 2) preamplifiers through isolated $+12 \mathrm{~V}$ and $-12 \mathrm{~V}$ bias lines with 
102

103

104

105

106

107

108

109

110

111

112

113

114

115

116

117

118

119

120

121

122

123

124

125

126

127

128

129

130

131

132

133

134

135

136

137

138

139

140

141

142

143

144

145

respect to earth ground. The imaging system operates at room temperature and no cooling systems were utilized for the LISe pixel detector or the front-end electronics. A single extruded aluminum enclosure encased the sensor board and front-end electronics, serving as an EMI and light shield. Aluminum is relatively transparent to cold neutrons, limiting neutron scattering and subsequent image degradation. The completed LISe pixel detector with the lid of the aluminum enclosure removed is presented in Fig. 3.

\subsection{Experimental}

The developed LISe pixel detector was tested at the High-Flux Isotope Reactor (HFIR) CG-1D Neutron Imaging Beamline at ORNL. The beam line exhibits an average neutron flux of $\sim 7 \times 10^{6} \mathrm{n} / \mathrm{cm}^{2} / \mathrm{s}$ between $0.8 \AA$ to $6 \AA$ with a peak at $\sim 2.6 \AA$ at the beam port $[18,19]$. The distance from the installed $\mathrm{ZnS}:{ }^{6} \mathrm{LiF}$ imaging plane and the point aperture used for these measurements was $6.6 \mathrm{~m}$ [20]. The LISe pixel detector was suspended on an aluminum extrusion approximately 0.3 meters in front of the $\mathrm{ZnS}:{ }^{6} \mathrm{LiF}$ scintillation screen (see Fig. 4).

Digital signal processing was carried out using two CAEN V1724 8-Channel Digitizers with on-board DPP-PHA processing interfaced to a PC via the A3818 PCI Express CONET2 Controller and CAEN V2718 bridge. The LISe pixel detector was biased at $250 \mathrm{~V}$ using a NHQ 203M Dual High Voltage Power Supply housed separately in an ORTEC 4001C NIM bin crate.

A 3/8 inch stainless steel bolt and PSI Siemens Star test pattern were used to test the LISe pixel detector [21]. The purpose of the bolt test was to image the thread pattern. The PSI mask has an outer diameter of $20 \mathrm{~mm}$ with $128 \mathrm{Gd}$ spokes (line/space pairs). From the innermost radius outwards, concentric rings provide visual pitch resolution markers between $40 \mu \mathrm{m}$ and $500 \mu \mathrm{m}$. A motorized stage was used to image objects larger than the $2.2 \times 2.2 \mathrm{~mm}^{2}$ active area of the LISe pixel detector via translation in the plane perpendicular to the cold neutron beam.

The LISe pixel detector was positioned along the centerline of the cold neutron beam where the flux approaches a maximum and is most uniform $[19,20]$. A custom LabVIEW ${ }^{\mathrm{TM}}$ program was created to automate bolt or PSI mask translation and data collection, where the measurement and translation stage movement times can be independently set to optimize exposure time and required sample translation time. This level of control allowed PSI mask translation below the pixel pitch of the LISe pixel sensor, now called the super-sampling technique, where the resolution of the LISe pixel detector is enhanced beyond the pixel pitch via data analysis techniques, as described in section 2.4. In Fig. 5, an image of the PSI mask and the range of positions investigated with the LISe pixel detector relative to the mask is provided. Here, the translation stage in the imaging sweep moved the PSI mask with a step size of 125 $\mu \mathrm{m}$ along 22 steps on both axes. A ten-second measurement time was used at each position with a two second movement time between measurement points.

\subsection{Simulation}

The experimental evaluation of the LISe pixel detector with the PSI mask was simulated to provide a benchmark for the experiment and provide an expected performance metric of the super-sampling technique. MCNP6 [22] was used to investigate a 4x4 LISe pixel detector with a pitch of $900 \mu \mathrm{m}$. The distance between the LISe pixel detector and the PSI mask was $15 \mathrm{~cm}$. The plane wave neutron source was emitted from a circular surface with a $5 \mathrm{~cm}$ radius at $25 \mathrm{meV}$ with zero divergence. Each simulation transported $10^{9}$ neutrons. The point spread function of the LISe pixel detector was ignored, a reasonable assumption for pixels of this size. The simulation step size was $50 \mu \mathrm{m}$ in a square pattern, 
146

147

148

149

150

151

152

153

154

155

156

157

158

159

160

161

162

163

164

165

166

167

168

169

170

171

172

173

174

175

176

177

178

179

180

181

182

183

resulting in 5,329 simulated positions orthogonal to the beam axis (z).

\subsection{Image Reconstruction}

For both experimental and computational results, a map was generated by organizing the counts in each pixel each position, $\mathrm{i}$ and $\mathrm{j}$, into a matrix $\phi_{c}(i, j)$. Utilizing the super-sampling technique, each pixel response matrix across the translation space are merged into a larger composite matrix, $\Phi$. The response matrices of each pixel are translated to reflect the expanded pixel map, $\phi_{c}^{\prime}$, where non-sensor pixel values are null when the pixel position is outside positions yielding experimental or computational results. The spatial locations where pixels overlap are averaged with the counting matrix, $A$.

$$
\Phi=\frac{1}{A} \sum_{c=1}^{16} \phi_{c}^{\prime}
$$

To facilitate the image reconstruction process from the experimental data, each measurement point was further divided into $25 \mu \mathrm{m}$ subsections in each dimension, helping align the individual channel images. Considering equation 1 , the values of $i$ and $j$ are redefined by the new sub-pixel spacing to $i^{\prime}$ and $j^{\prime}$, decreasing both by a sub-pixel factor, $f_{s}=5$. The expansion generates each new sub-pixel of the intensity map, $I_{c}$, with an $f_{s} \times f_{s}$ matrix,

$$
I_{c}^{\prime}\left(i^{\prime}, j^{\prime}\right)=\left[\frac{\phi_{c}(i, j)}{f_{s}^{2}}\right]_{f_{s} \times f_{s}}
$$

where $i^{\prime}=\left[1,2,3, \ldots f_{s}\right] \cdot i$ and $j^{\prime}=\left[1,2,3, \ldots f_{s}\right] \cdot j$. From here, the individual channel intensity maps are meshed using a modified version of equation 1 , given in equation 3 . Similar to the expanded neutron intensity map (equation 2), the expanded pixel map, $I_{c}^{\prime}$, has been translated to accommodate the full sensor pattern, where the similar sized matrix, $\alpha$, functions as the average counting matrix. Prenormalizing each pixel before image reconstruction enhances the final matrix, $I_{\text {image. }}$. The final output images scale a unit range, corresponding to either zero or maximum measured neutron intensity [23, 24].

$$
I_{\text {image }}=\frac{1}{\alpha} \sum_{c=1}^{16} I_{c}^{\prime}
$$

\section{RESUlts}

\subsection{Neutron Radiography}

From the MCNP simulations, the $400 \mu \mathrm{m}$ and $500 \mu \mathrm{m}$ bands and perpendicular line pairs of the Siemens star are clearly visible (see Fig. 6). To compare the results from multiple experimental setups, it is helpful to define a resolution quality factor, $f_{R}$, as the quotient of the pitch size, $D_{P}$, and the minimum resolvable feature size, $L_{\mathrm{min}}$. 
184

185

186

187

188

189

190

191

192

193

194

195

196

197

198

199

200

201

202

203

204

205

206

207

208

209

210

211

212

213

214

215

216

217

218

219

220

221

222

223

224

225

226

227

228

229

230

$$
f_{R}=\frac{D_{P}}{L_{\min }}
$$

The MCNP simulation produced a value of $f_{R}=2.25$, demonstrating the capability of resolving features less than one-half the pixel pitch. This result provides an ideal neutron transmission radiograph for a LISe pixel detector with a $900 \mu \mathrm{m}$ pixel pitch from a homogeneous neutron plane wave with zero divergence, indicating the capability of the super-sampling technique with a step size of $50 \mu \mathrm{m}$.

Experimental evaluation of the LISe pixel sensor utilized a 10 second integration at each spatial position across the outer regions of the PSI Siemens Star. The resulting neutron radiograph is provided in Fig. 7, which shows the 500, 400 and $300 \mu \mathrm{m}$ concentric pitch markers, leading to a maximum experimental resolution factor of $f_{R}=1.83$. While the experimental value is less than the simulation, the result implies that the obtainable resolution may be higher than reported if the experiment would have investigated farther into the center of the Siemens star.

The neutron radiograph of the bolt generated with the LISe pixel detector is provided in Fig. 8 and overlaid on a neutron radiograph taken with the RC TRITEC ${ }^{6} \mathrm{LiF}: \mathrm{ZnS}(2: 1)$ scintillation screen. The color scale bar provided follows the neutron intensity, where green is high neutron response and yellow is low neutron response. The three black regions on the scale bar was found necessary to show the required contrast, providing scale bar limits and the transition between low (yellow) and high (green) neutron response. During the 12-hour experimental run, the average neutron count rate per channel decreased by approximately $20 \%$ at an estimated neutron fluence of $\sim 4 \times 10^{11}$ neutrons $/ \mathrm{cm}^{2}$. However, identification of the bolt threads was unaffected by the measured count rate change and the image contrast is comparable to the ${ }^{6} \mathrm{LiF}: \mathrm{ZnS}$ scintillator screen. Still, a $20 \%$ decrease in average count rate across the LISe pixel detector for this fluence was not expected, so further investigation was conducted.

\subsection{Pixel Response}

The background and open beam flat field count rate were recorded at the start and end of experimentation to calculate the neutron count rate (see Fig. 9). Dividing the neutron detection rate by the average incident neutron rate gives an estimate of the total system detection efficiency. The anticipated efficiency of the LISe pixel detector at a $3.927 \AA$ average neutron wavelength is $59 \%$ for the ${ }^{6} \mathrm{Li}\left(\mathrm{n},{ }^{3} \mathrm{H}\right){ }^{4} \mathrm{He}$ reaction. In the experiment, the best performing pixels exhibited a neutron detection efficiency between $35 \%$ and $55 \%$.

The reduced and varying detection efficiency measured is attributed to variations in the individually chosen threshold pedestal discrimination levels for each pixel and material inhomogeneities (inclusions and trapping centers). Even for the best performing pixels, the neutron detection efficiency did to reach $59 \%$, which is due to the poor hole carrier properties. For point-of-fact, Fig. 10 shows the response of a planar LISe sensor to ${ }^{234} \mathrm{U}$ alpha particles, ${ }^{137} \mathrm{Cs}$ gamma-rays, and moderated ${ }^{239} \mathrm{Pu}$-Be neutrons to demonstrate the hole transport properties of LISe. For the ${ }^{234} \mathrm{U}$ alpha particle exposure, a peak is only observed for electron collection, but counts are observed when collecting holes as well. With the provided response of the planar LISe sensor to moderated ${ }^{239} \mathrm{Pu}-\mathrm{Be}$ neutron response given in Fig. 10, we can see that holes do contribute to the measured neutron response, where the shelf in the neutron response resides at a higher channel number above the peak observed from ${ }^{234} \mathrm{U}$ alpha particles $(\sim 4.77$ $\mathrm{MeV})$. The broad distribution of the neutron response is also characteristic of poor charge transport properties, primarily due to holes. For the LISe pixel sensor, one collected background and neutron spectra for channel one is given in Fig. 11. A small and broad peak in the spectrum is observed followed by a long tail. The size of the pixels is on the order of the thickness of the LISe substrate, so 
231

232

233

234

235

236

237

238

239

240

241

242

243

244

245

246

247

248

249

250

251

252

253

254

255

256

257

258

259

260

261

262

263

264

265

266

267

268

269

270

271

272

273

274

275

276

277

278

279

variations in a linear weighting potential is likely the reason for the difference observed in the neutron response between Fig. 10 and Fig. 11 [25].

The drop in the count rate after the 12-hour exposure during imaging of the bolt is expected to be due to a combination of radiation damage and the sensor architecture. The front region of the detector (positively biased) will experience the most damage via the charged particles from the ${ }^{6} \mathrm{Li}\left(\mathrm{n},{ }^{3} \mathrm{H}\right){ }^{4} \mathrm{He}$ reaction, consistent with first order transport detection efficiency. The low hole charge carrier properties and high damage rate at the front contact of the LISe pixel detector is likely the cause of the lowered sensitivity over the 12-hour neutron exposure.

\section{Conclusion}

The potential of the LISe pixel detector for neutron radiographic imaging has been presented. The proof-of-principle sensor provides promise for high spatial resolution neutron radiography. The spatial resolution of the LISe pixel detector has been confirmed at sub pixel size, achieving a resolution of 300 $\mu \mathrm{m}$ with a pixel pitch of $550 \mu \mathrm{m}$. The use of super-sampling to achieve this spatial resolution agrees with the simulated response of a LISe pixel detector. The pixel neutron detection efficiency varied significantly between pixels, likely beyond expected variations due to signal processing electronics. Reduction in crystal defects and inclusions via enhanced LISe growth should help to normalize the response across pixels. Reduction in the neutron count rate can be minimized by switching the polarity of the device, collecting holes at the front contact rather than the back contact.

The potential spatial resolution of a LISe pixel sensor is limited by the $47 \mu \mathrm{m}$ combined range of secondary charged particles in LISe. The $4 \pi$ emission of these secondary charged particles suggests that a higher spatial resolution is possible beyond $47 \mu \mathrm{m}$ with current ASIC technology [26], albeit with some potential broadening of the line spread function. As integrated circuitry advances, secondary charged particle tracking and advanced signal processing could lead to a much higher spatial resolution.

\section{ACKNOWLEDGEMENTS}

This work is supported through subcontract number 4300090406 from CNS Y-12 National Security Complex and under a Department of Energy Nuclear Energy University Programs Graduate Fellowship. A portion of this research at ORNL's High Flux Isotope Reactor was sponsored by the Scientific User Facilities Division, Office of Basic Energy Sciences, U.S. Department of Energy.

\section{REFERENCES}

[1] I. S. Anderson, R. L. McGreevy, and H. Z. Bilheux, Neutron Imaging and Applications. New York, NY: Springer, 2009.

[2] H. E. Martz, C. M. Logan, D. J. Schneberk, and P. J. Shull, X-Ray Imaging: Fundamentals, Industrial Techniques, and Applications: Taylor \& Francis, 2016.

[3] A. A. Harms and D. R. Wyman, Mathematics and physics of neutron radiography vol. 1: Springer Science \& Business Media, 1986.

[4] G. Carini, P. Denes, and S. Gruner, "Neutron and X-ray Detectors: Report of the Basic Energy Sciences Workshop on Neutron and X-ray Detectors," DOE Office of Science2012.

[5] J. Jakubek, T. Holy, E. Lehmann, S. Pospisil, J. Uher, J. Vacik, et al., "Neutron imaging with Medipix-2 chip and a coated sensor," Nuclear Instruments and Methods in Physics Research Section A: Accelerators, Spectrometers, Detectors and Associated Equipment, vol. 560, pp. 143147, May 12006. 
[6] D. S. McGregor, J. T. Lindsay, C. C. Brannon, and R. W. Olsen, "Semi-insulating bulk GaAs thermal neutron imaging arrays," IEEE Transactions on Nuclear Science, vol. 43, pp. 13571364, 1996.

[7] D. S. McGregor, M. D. Hammig, Y. H. Yang, H. K. Gersch, and R. T. Klann, "Design considerations for thin film coated semiconductor thermal neutron detectors-I: basics regarding alpha particle emitting neutron reactive films," Nuclear Instruments and Methods in Physics Research Section A: Accelerators, Spectrometers, Detectors and Associated Equipment, vol. 500, pp. 272-308, 3/11/ 2003.

[8] G. Bruckner, A. Czermak, H. Rauch, and P. Weilhammer, "Position sensitive detection of thermal neutrons with solid state detectors (Gd Si planar detectors)," Nuclear Instruments and Methods in Physics Research Section A: Accelerators, Spectrometers, Detectors and Associated Equipment, vol. 424, pp. 183-189, 11/3/ 1999.

[9] P. Trtik and E. H. Lehmann, "Isotopically-enriched gadolinium-157 oxysulfide scintillator screens for the high-resolution neutron imaging," Nuclear Instruments and Methods in Physics Research Section A: Accelerators, Spectrometers, Detectors and Associated Equipment, vol. 788, pp. 67-70, 7/11/ 2015.

[10] M. Strobl, I. Manke, N. Kardjilov, A. Hilger, M. Dawson, and J. Banhart, "Advances in neutron radiography and tomography," Journal of Physics D: Applied Physics, vol. 42, p. 243001, 2009.

[11] A. S. Tremsin, J. B. McPhate, J. V. Vallerga, O. H. W. Seigmund, and W. B. Feller, "Improved efficiency of high resolution thermal and cold neutron imaging," Nuclear Instruments and Methods in Physics Research A, vol. 628, pp. 415-418, 2011.

[12] J. Banhart, Advanced Tomographic Methods in Materials Research and Engineering: Oxford University Press, 2008.

[13] E. Tupitsyn, P. Bhattacharya, E. Rowe, L. Matei, Y. Cui, V. Buliga, et al., "Lithium containing chalcogenide single crystals for neutron detection," Journal of Crystal Growth, vol. 393, May 1, 2014.

[14] B. Wiggins, E. Tupitsyn, P. Bhattacharya, E. Rowe, E. Lukosi, O. Chvala, et al., "Investigation of non-uniformity and inclusions in 6LiInSe2 utilizing laser induced breakdown spectroscopy (LIBS)," in SPIE, San Diego, CA, 2013, p. 88520M.

[15] E. D. Lukosi. (2015, Aug 17). JIAM Micro-Processing Research Facility. Available: http://jiam.utk.edu/facilities/mprf/index.php

[16] S. A. Campbell, Fabrication Engineering at the Micro- and Nanoscale, 3rd ed.: Oxford University Press, 2008.

[17] L. Rossi, P. Fischer, T. Rohe, and N. Wermes, Pixel Detectors, 2006.

[18] H. Z. Bilheux, J.-C. Bilheux, W. B. Bailey, W. S. Keener, L. E. Davis, K. W. Herwig, et al., "Neutron imaging at the Oak Ridge National Laboratory: Application to biological research," in Biomedical Science and Engineering Center Conference (BSEC), 2014 Annual Oak Ridge National Laboratory, 2014, pp. 1-4.

[19] L. Crow, L. Robertson, H. Bilheux, M. Fleenor, E. Iverson, X. Tong, et al., "The CG1 instrument development test station at the high flux isotope reactor," Nuclear Instruments and Methods in Physics Research Section A: Accelerators, Spectrometers, Detectors and Associated Equipment, vol. 634, pp. S71-S74, 4/1/ 2011.

[20] L. Santodonato, H. Bilheux, B. Bailey, J. Bilheux, P. Nguyen, A. Tremsin, et al., "The CG-1D Neutron Imaging Beamline at the Oak Ridge National Laboratory High Flux Isotope Reactor," Physics Procedia, vol. 69, pp. 104-108, // 2015.

[21] C. Grunzweig, G. Frei, E. Lehmann, G. Kuhne, and C. David, "Highly absorbing gadolinium test device to characterize the performance of neutron imaging detector systems," Review of Scientific Instruments, vol. 78, pp. 053708-053708-4, 2007.

[22] MCNP6 User's Manual, Version 1.0, Denis Pelowitz et al., Los Alamos, NM, 2008. 
329

330

331

332

333

334

335

336

337

338

339

340
[23] S. A. Dianat and E. Saber, Advanced Linear Algebra for Engineers with MATLAB, 1st ed. Boca Raton, FL: CRC Press, 2009.

[24] F. Y. Shih, Image Processing and Pattern Recognition:Fundamentals and Techniques: WileyIEEE Press, 2010.

[25] R. H. den Hartog, A. Owens, A. G. Kozorezov, M. Bavdaz, A. J. Peacock, V. Gostilo, et al., "Optimization of array design for TIBr imaging detectors," 2003, pp. 922-932.

[26] X. Llopart, R. Ballabriga, M. Campbell, L. Tlustos, and W. Wong, "Timepix, a 65k programmable pixel readout chip for arrival time, energy and/or photon counting measurements," Nuclear Instruments and Methods in Physics Research Section A: Accelerators, Spectrometers, Detectors and Associated Equipment, vol. 581, pp. 485-494, Oct 212007. 
Fig. 1. Two semiconducting LISe crystals ( $7 \mathrm{~mm}$ largest dimension).

Fig. 2. Photolithographic processing steps using negative resist to facilitate metal lift-off. Step (1) chemical/mechanical polish, (2) spin-coat negative photoresist, (3) mask and UV exposure, (4) develop pattern, (5) deposit metallic contacts and (6) lift-off excess metal.

Fig. 3. LISe pixel detector patterned with gold in 16-channel array (left) and LISe pixel detector with top of enclosure removed (right).

Fig. 4 . High Flux Isotope Reactor CG-1D beam line experimental setup.

Fig. 5. PSI mask optical microscope image (left) [20] and experimental interrogation area of the LISe pixel detector (right).

Fig. 6. MCNP simulated response of LISe pixel detector (900 $\mu \mathrm{m}$ pixel pitch) with the PSI Siemens Star [20], utilizing the super sampling technique (50 $\mu \mathrm{m}$ steps).

Fig. 7. Reconstructed image of the PSI Siemens Star [20] using the developed LISe pixel detector (550 $\mu \mathrm{m}$ pixel pitch) and the super sampling technique (125 $\mu \mathrm{m}$ steps).

Fig. 8. Image of a stainless steel bolt generated with the LISe pixel detector overlaid on the radiograph taken with the RC TRITEC $50 \mu \mathrm{m} \mathrm{ZnS:}{ }^{6} \mathrm{LiF}(2: 1)$ scintillation screen. Image is normalized to the mean white field.

Fig. 9. Detection efficiency across the LISe pixel detector array before and after experimentation.

Fig. 10. Response of a planar LISe detector to Cs-137 gamma-rays, ${ }^{234} \mathrm{U}$ alpha particles (negative bias is indicated in the legend), and ${ }^{239} \mathrm{Pu}-\mathrm{Be}$ neutrons and associated $2.1 \mathrm{MeV}$ and 4.6 MeV gamma-rays.

Fig. 11. Response of a single pixel of the LISe pixel detector to the cold neutrons at the CG-1D Neutron Imaging Beam Line at ORNL. 


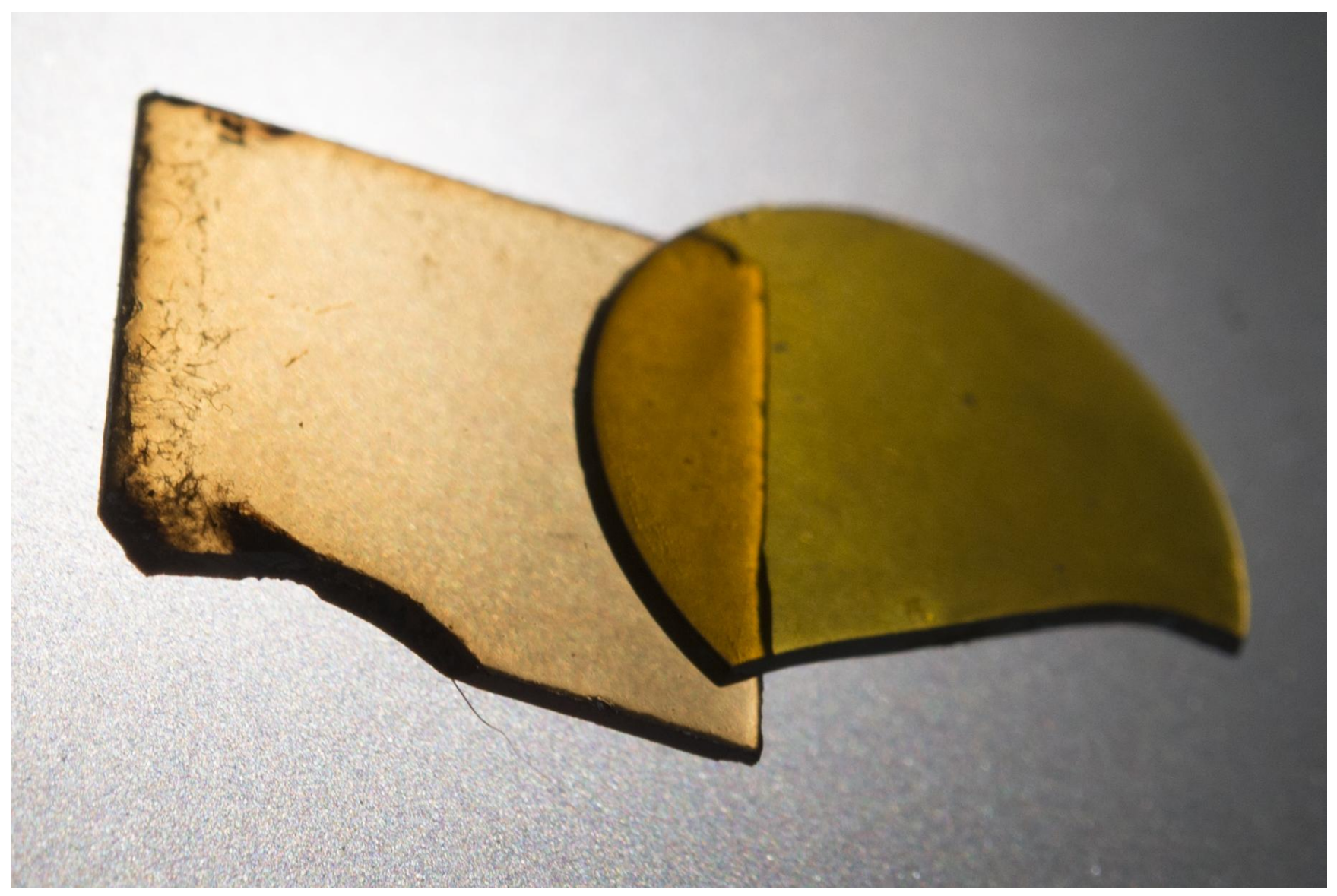



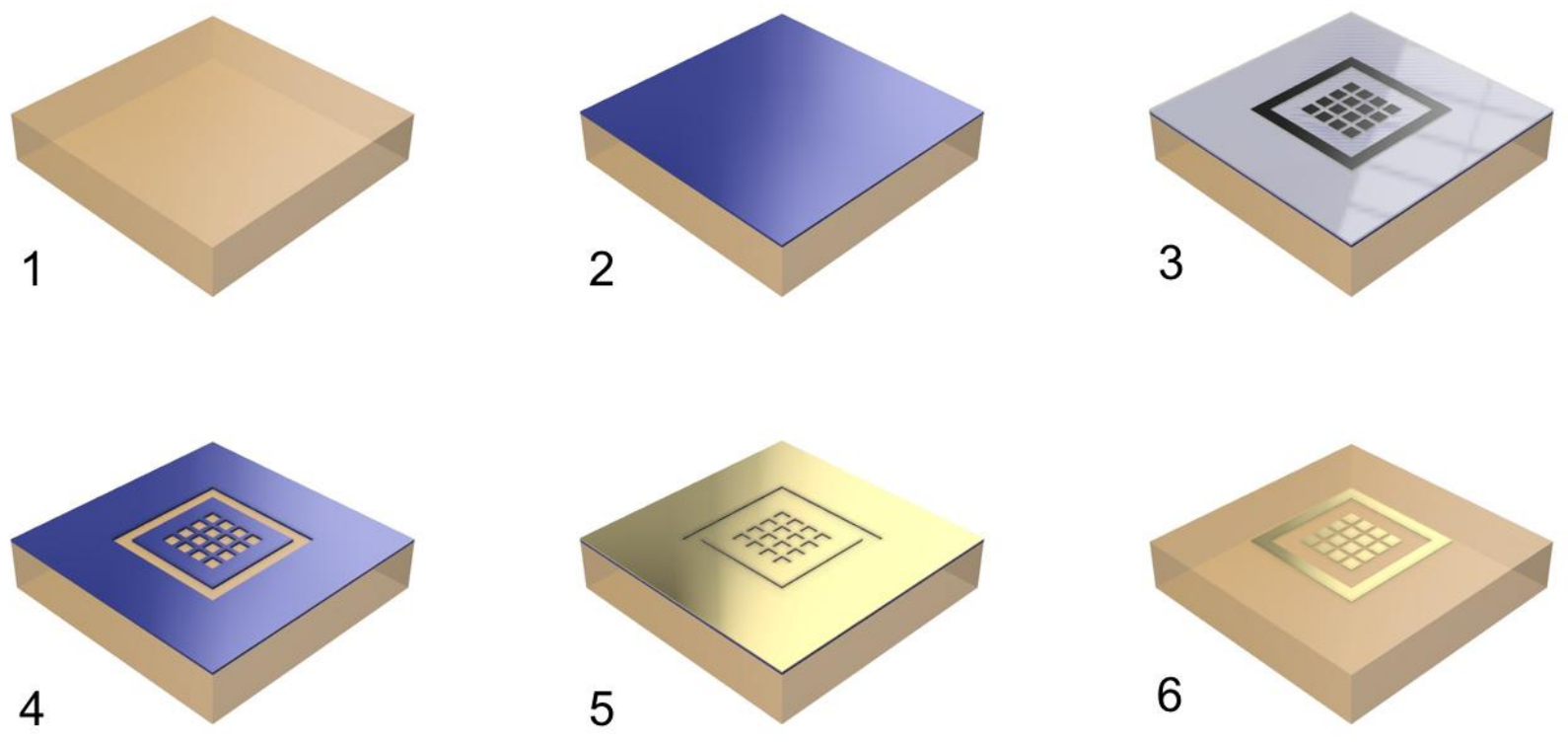


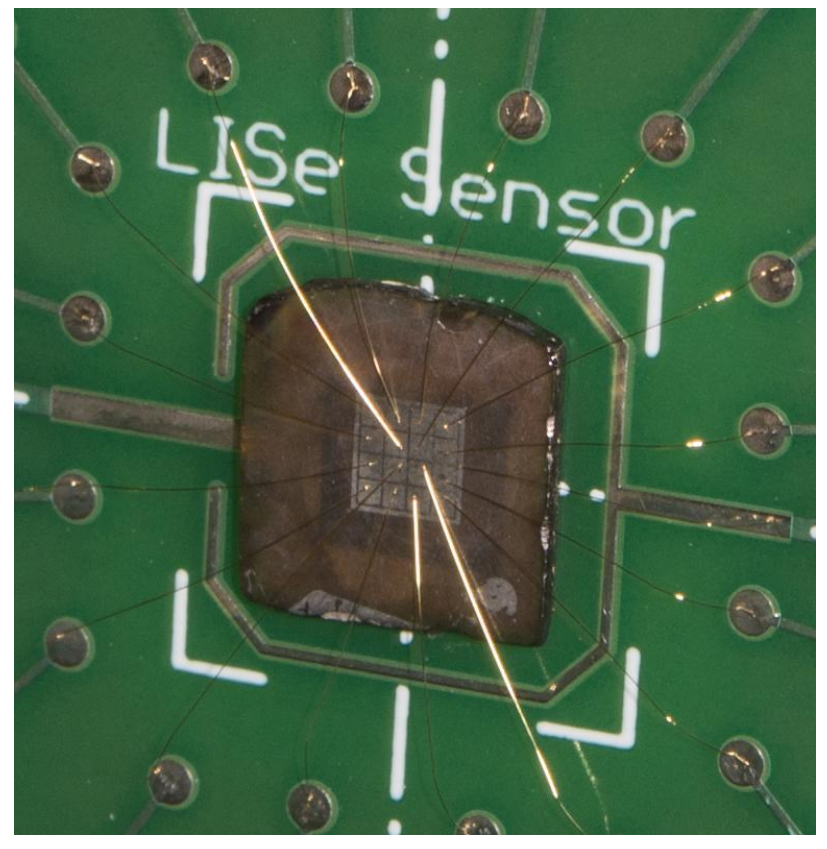


13

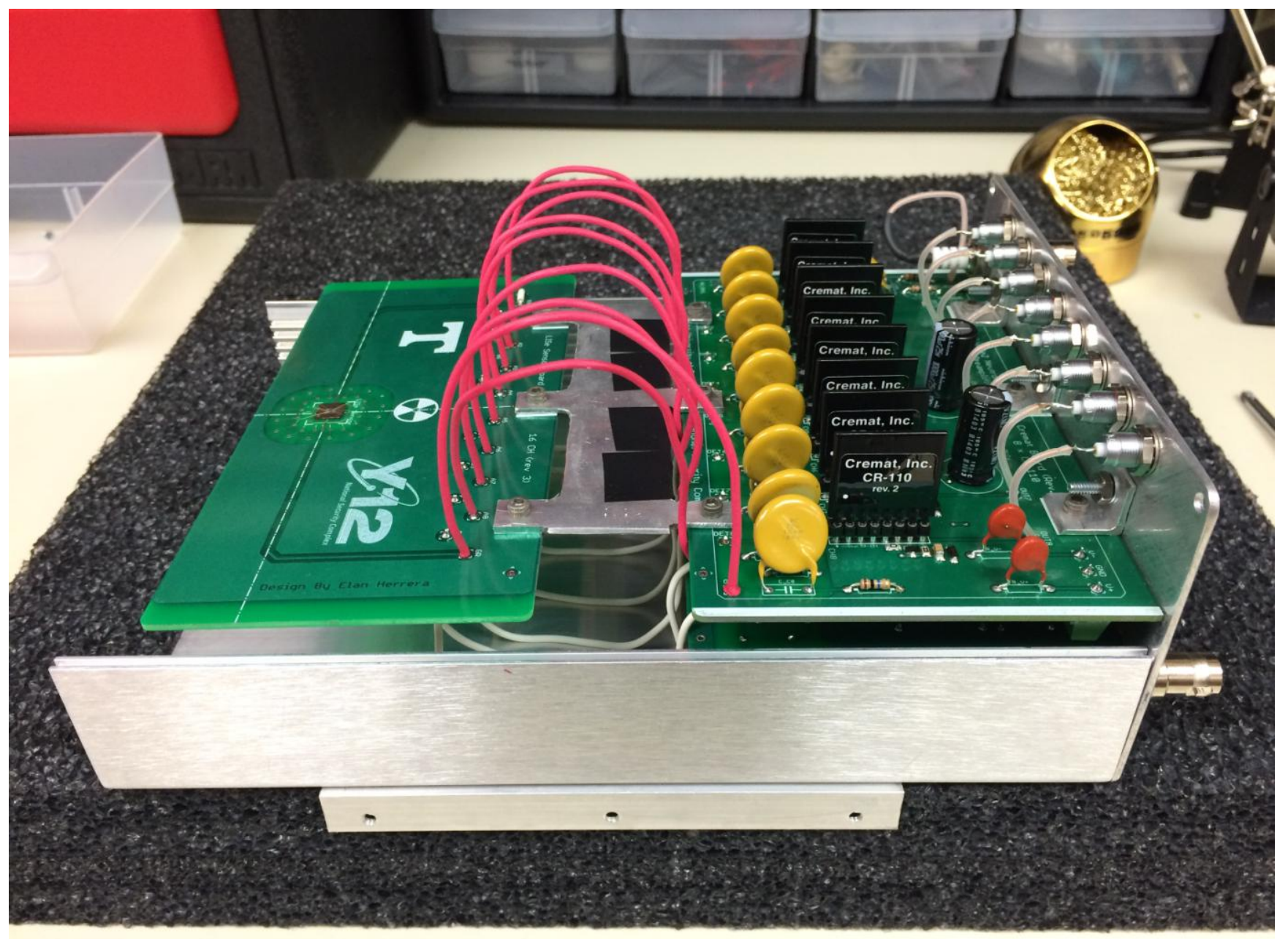




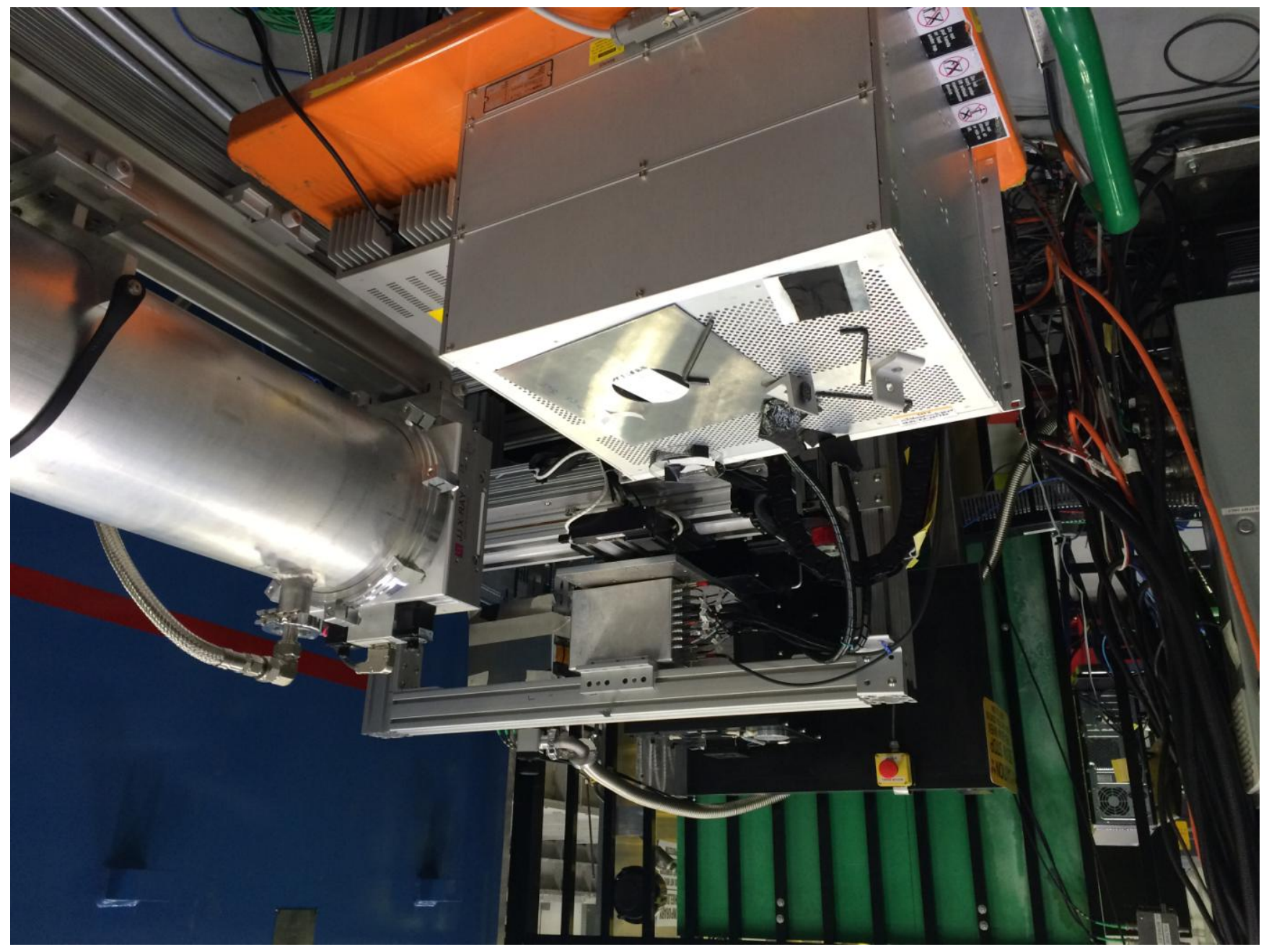




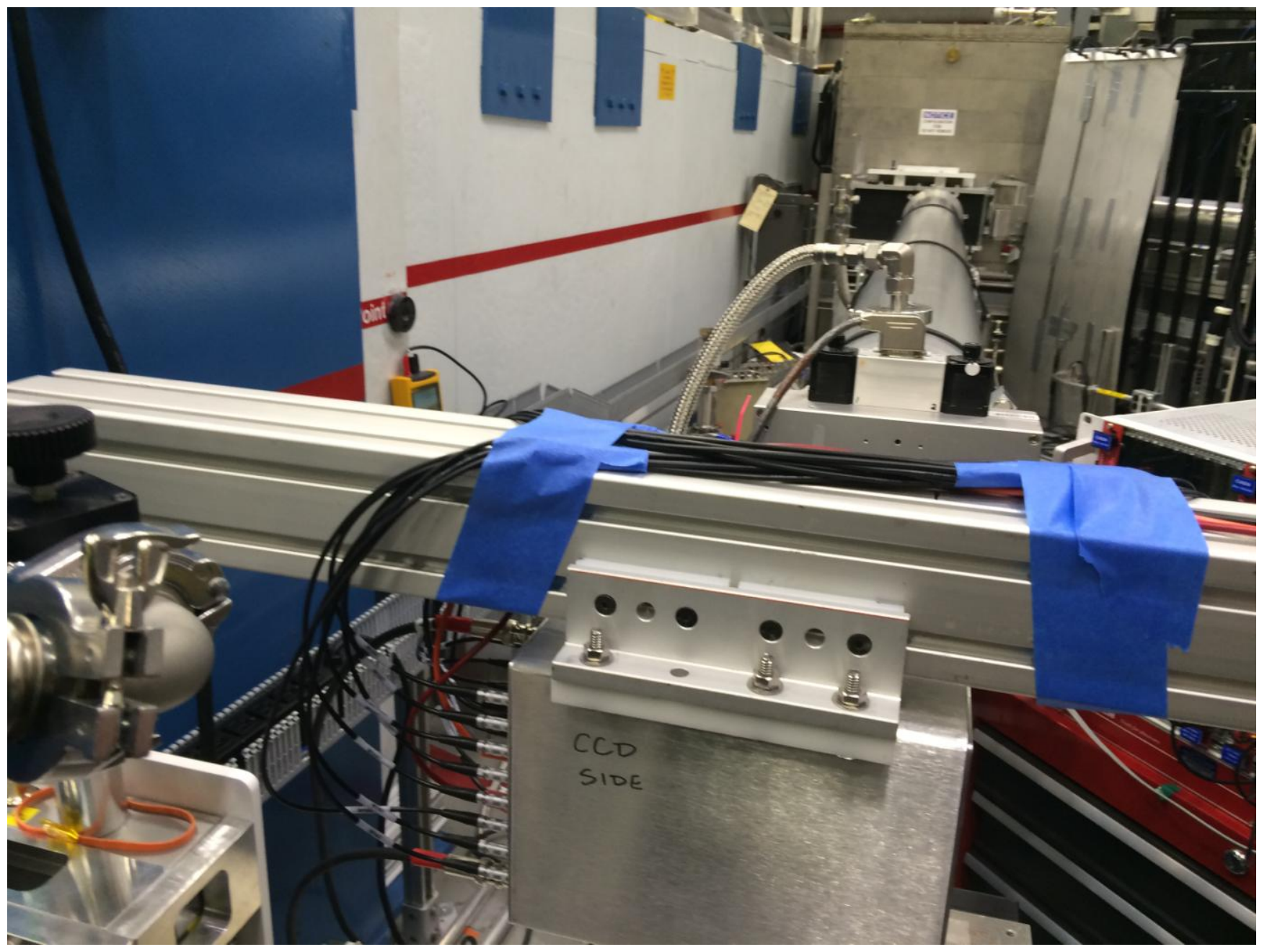




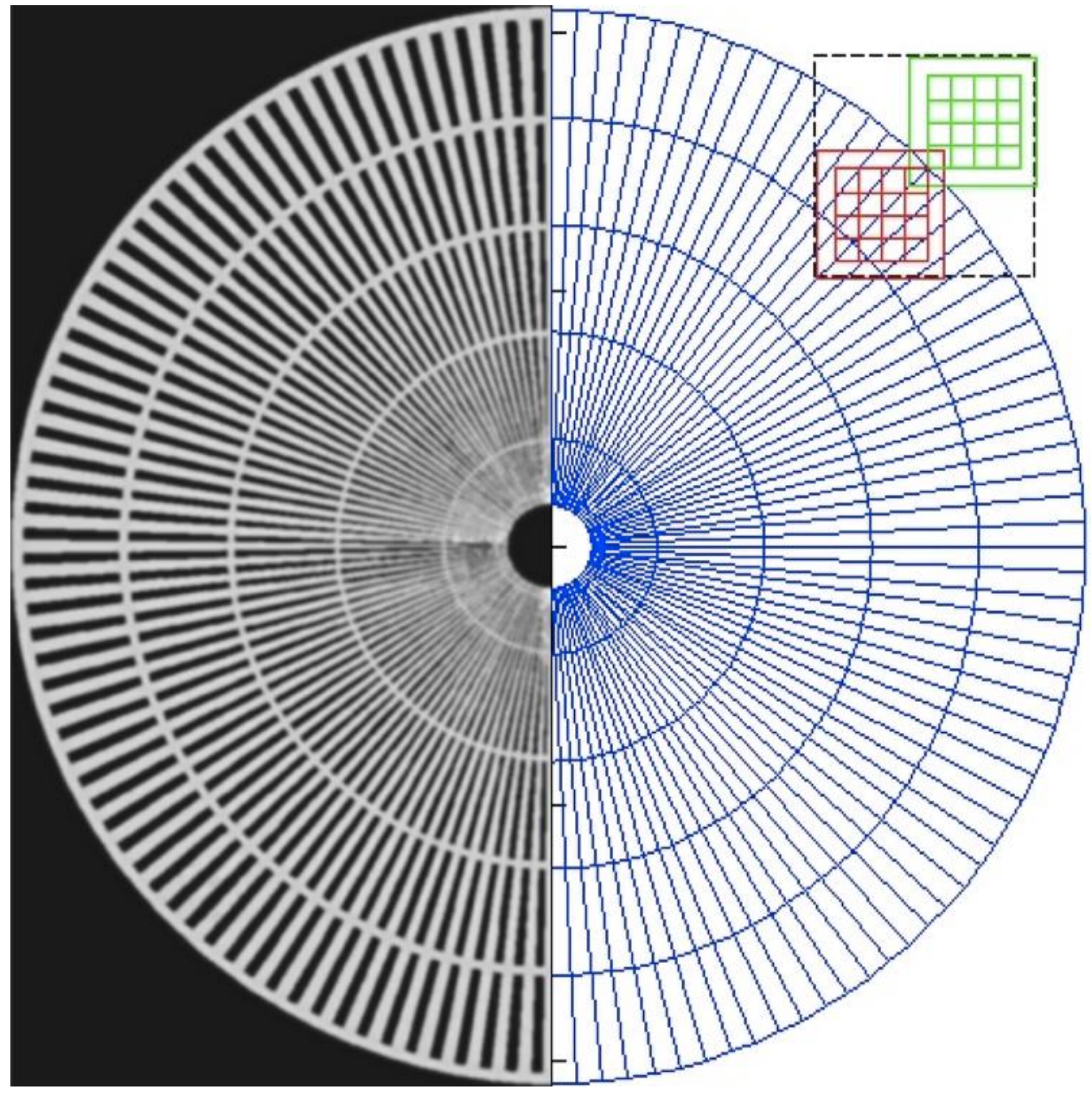




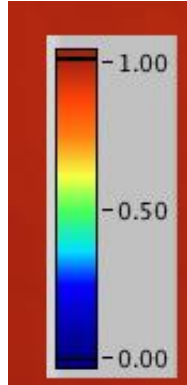




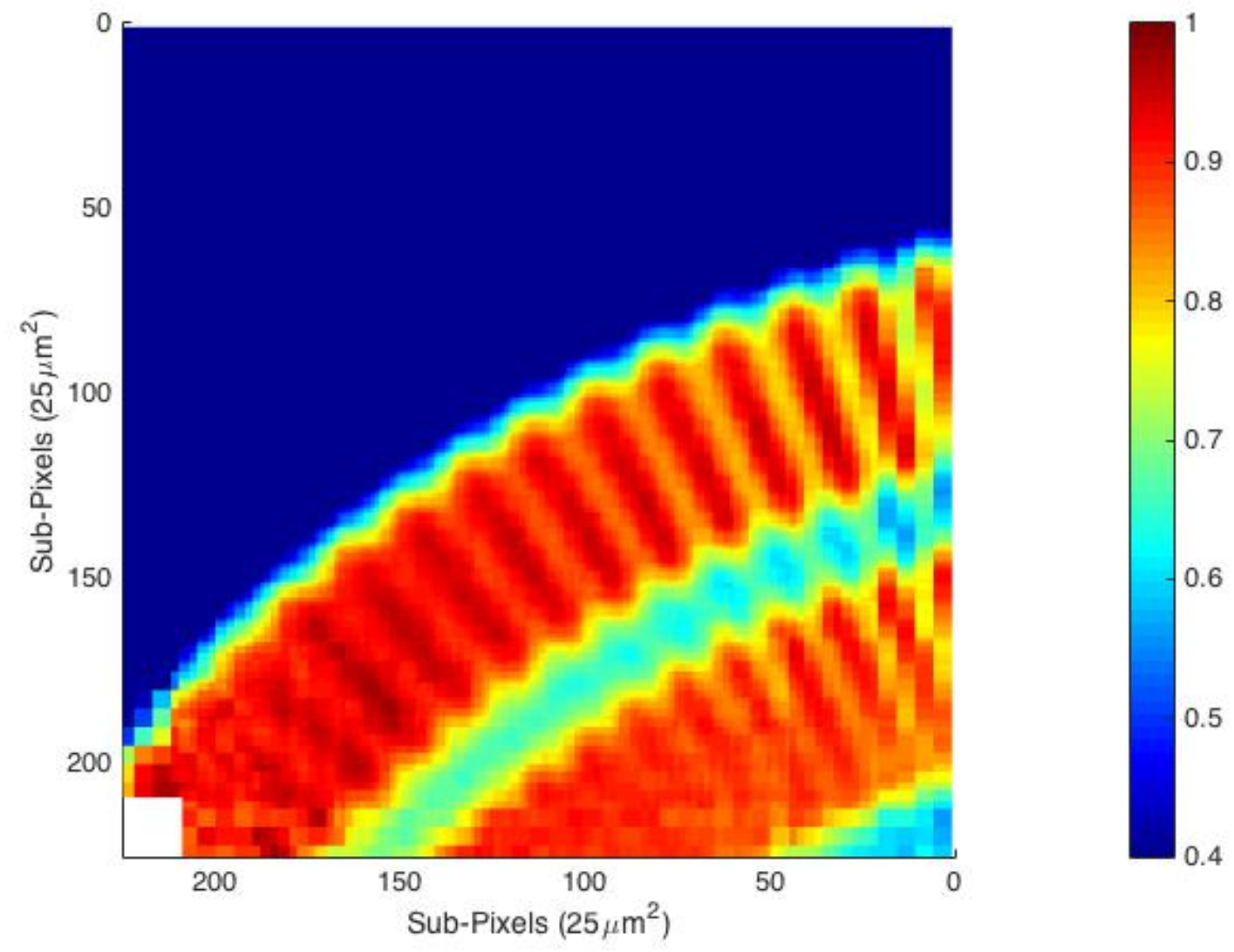



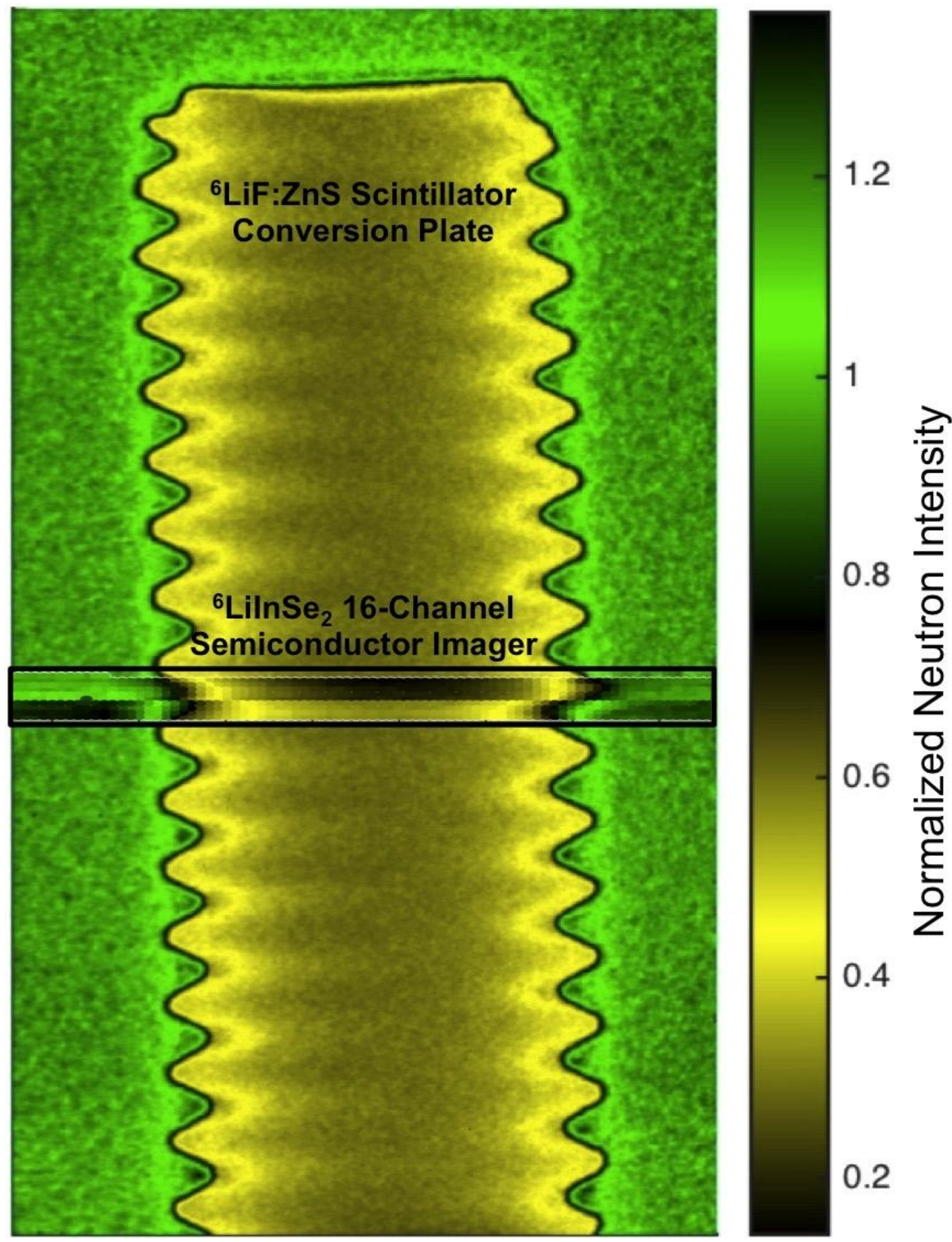


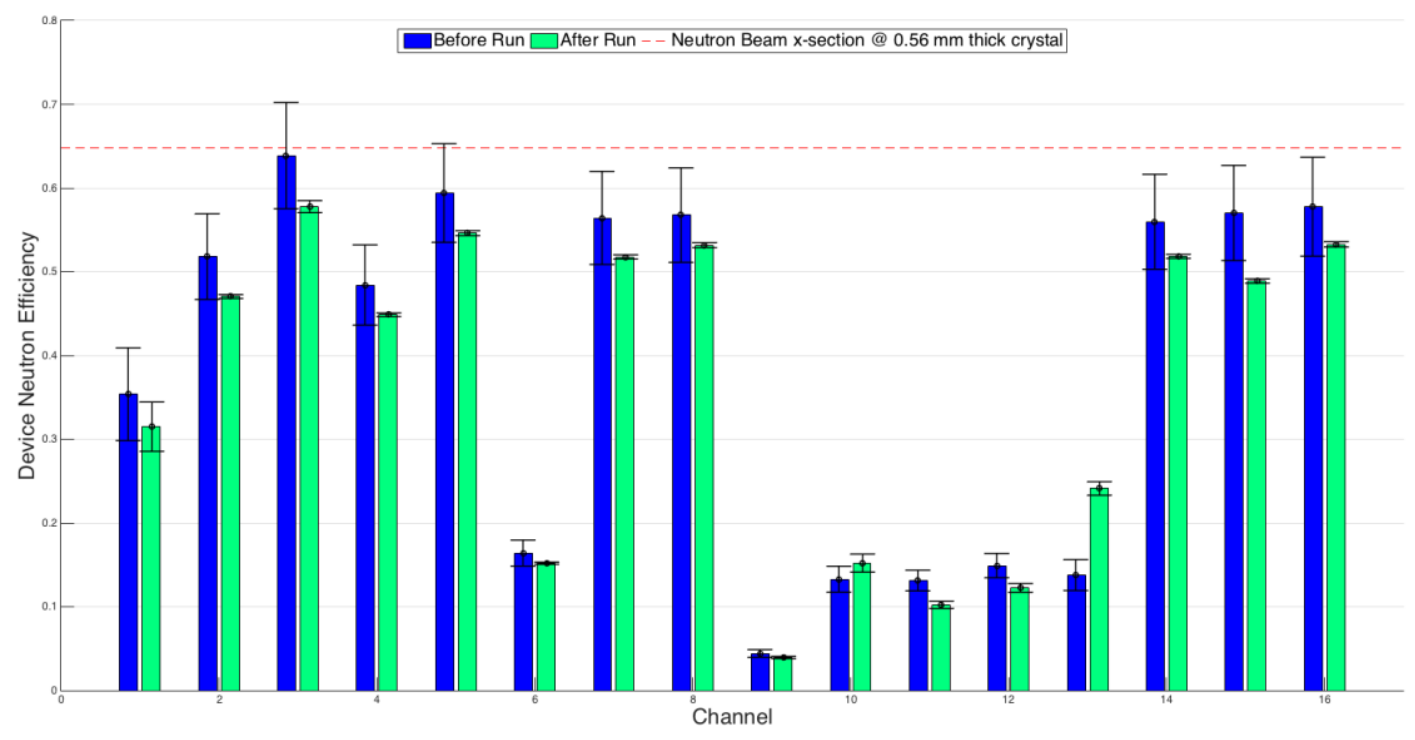




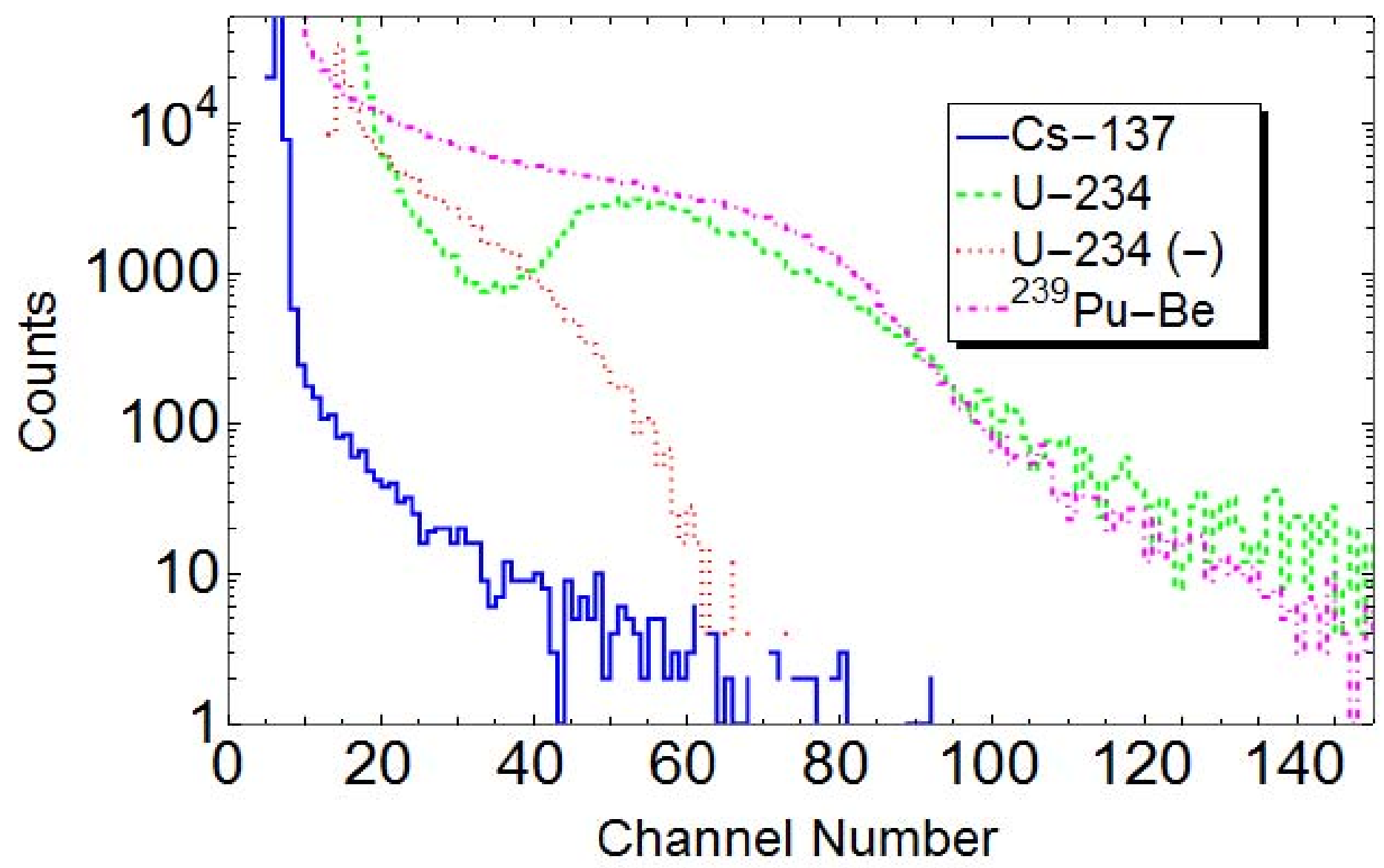




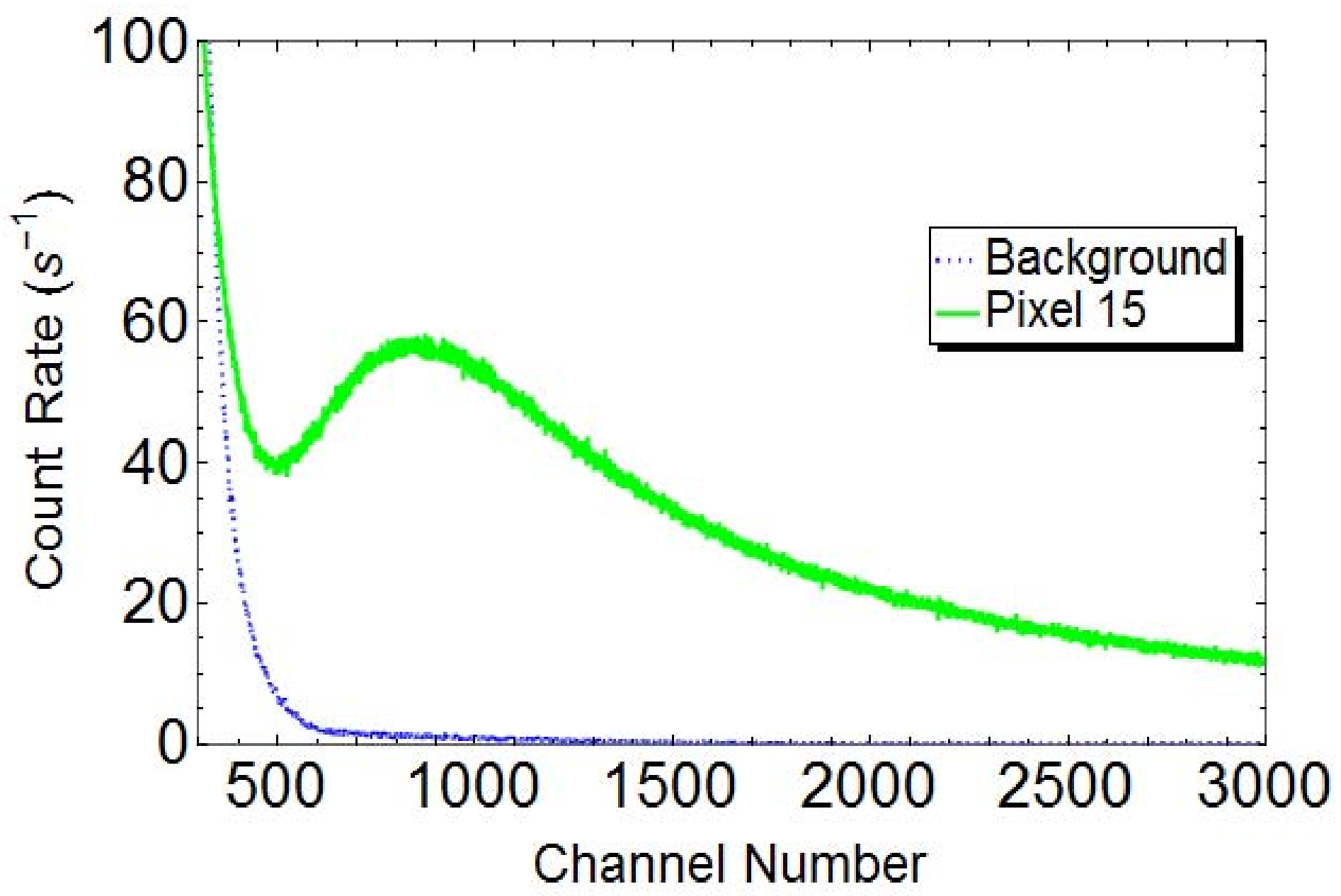

